\title{
Emociones positivas y marketing social en el Facebook de las ONGDs
}

\author{
Positive emotions and social marketing on NGDO Facebook pages
}

\author{
Dra. Araceli Galiano Coronil \\ Universidad de Cádiz | Avda. de la Universidad, 4 - 11406 Jerez de la Frontera (Cádiz) | España \\ | https://orcid.org/0000-0003-2270-0924|araceli.galiano@gm.uca.es
}

\author{
Dr. Rafael Ravina Ripoll \\ Universidad de Cádiz | Avda. de la Universidad, 4 - 11406 Jerez de la Frontera (Cádiz) | España \\ | https://orcid.org/0000-0001-7007-3123|rafael.ravina@uca.es
}

Fechas | Recepción: 21/01/2021| Aceptación: 24/04/2021

Resumen

Existe un gran interés por la comunidad académica en medir las emociones positivas. Bajo este paraguas, surge una importante literatura que empiezan a explorar estas emociones en el entorno del voluntariado de las ONGDs. Estas entidades emplean las redes sociales para comunicarse con la ciudadanía, siendo Facebook una de las más usadas ya que permite establecer una comunicación bidireccional con los usuarios. Desde esta perspectiva, el presente artículo quiere examinar la influencia de las emociones positivas y el marketing social en los mensajes que hablan de voluntariado a partir de la información proporcionada por los mensajes publicados en las 10 ONGDs más populares en Facebook durante el año 2019. Con este propósito se ha utilizado una metodología basada en minería de texto y análisis de contenido desde un enfoque cuantitativo $y$ cualitativo. Para ello se usaron los programas informáticos SPSS V.27 y Nvivo 12 plus. Los resultados muestran que el número de likes está asociado con la aparición de términos sobre emociones positivas en los mensajes que hablan de voluntariado. También hay determinados vocablos con connotaciones positivas que son los más publicados por las

\begin{abstract}
There is great interest in the academic community in measuring positive emotions. In this sense, important literature emerges that explores these emotions in the volunteer environment of NGDOs. These entities use social networks to communicate with citizens, Facebook being one of the most used since it allows establishing bidirectional communication with users. From this perspective, this article wants to examine the influence of positive emotions and social marketing in messages that talk about volunteering, from the information provided by the messages published in the ten most popular NGDOs on Facebook during the year 2019. For this purpose, a methodology based on text mining and content analysis from a quantitative and qualitative approach was used. For this purpose, SPSS V.27 and Nvivo 12 plus software were used. The results show that the number of likes is associated with the appearance of terms synonymous with happiness in the messages that talk about volunteering. There are also certain words with positive connotations that are the most published by organizations under study. From the point of view of social marketing, informative messages have achieved more
\end{abstract}


organizaciones. Desde el punto de vista del impact than behavioral ones. marketing social, destaca el impacto conseguido por los mensajes informativos y el escaso impacto de los mensajes de comportamiento.

Palabras clave: marketing social, felicidad, ONGD, Facebook.

Keywords: social marketing, happiness, NGOD, Facebook.

\section{INTRODUCCIÓN}

No es difícil encontrar una vasta información sobre la economía de la felicidad desde finales del siglo XX hasta la actualidad. Dicho fenómeno viene originado por múltiples causas, entre ellas, cabe citar, la creación del Índice global de la felicidad por parte de la Organización de Naciones Unidas (ONU), y la entrega del Premio Nobel de Economía a Joseph Stiglizt (2001) por considerar a la felicidad como uno de los recursos intangibles más útiles que poseen los gobiernos para medir a nivel macroeconómico la calidad de vida y el bienestar colectivo de la sociedad civil (Tanaka y Tokimatsu, 2020). En este contexto el marketing social se configura como una relevante disciplina, ya que su fin último es influir en el comportamiento voluntario de la audiencia objetivo para mejorar su bienestar personal y el de su sociedad (Andreasen, 1994; French \& Blair, 2006; Dann, 2010; Kotler, 2011).

En relación a lo anterior, cabe señalar que son pocos los estudios académicos que investigan el grado de influencia directa que poseen los roles solidaridad, generosidad y altruismo sobre la felicidad de las personas (Schwarze \& Winkelmann, 2011). De ahí, la necesidad de analizar las citadas variables sobre la felicidad de los ciudadanos que desarrollan de forma habitual actividades o tareas de voluntariado en las Organizaciones No Gubernamentales para el Desarrrollo (ONGDs). Cuestión que poco se sabe ante la falta de un panorama estadístico internacional que contemple dicha información, la ausencia de modelos ecnométricos que evalúen la felicidad de los voluntarios en la sociedad digital (Borgonovi, 2008) y la efectividad de herramientas actuales como son las redes sociales como instrumenos de marketing social para conseguir el bienestar en el mencionado colectivo. De ahí la dificultad de considerar la felicidad de este citado capital humano como un elemento que contribuye holísticamente a construir una sociedad más justa, igualitaria y humanista (Ravina-Ripoll et al., 2018).

A resulta de lo anterior, el presente artículo nace con el objetivo básico de investigar el binomio marketing social-felicidad, con la finalidad de conocer en qué medida los mensajes de Facebook de las organizaciones objeto de estudio hablan de voluntariado y felicidad. Explorar la reacción del público ante estos mensajes, conocer sus características desde la perspectiva del marketing social, y saber si existe relación entre la reacción del público y el contenido de felicidad de las publicaciones.

\section{MARCO TEÓRICO}

Desde mediados de los años setenta del siglo pasado, los economistas y los sociólogos empiezan a interesarse de forma activa por medir el recurso intangible de la felicidad, siendo conscientes de que este término tiene importantes connotaciones subjetivas $y$, por ende, psicológicas (ej. Ashtiani et al., 2020; Bakker et al.,2020; Crespo \& Mesurado,2015). De este modo surge la economía de la felicidad, disciplina que se encuadra dentro del área de la 
economía del bienestar con la finalidad de demostrar, entre otras cosas, como los factores sociodemográficos (edad, género, rentas, nivel de estudios, etc.) y psico organizacionales (clima organizacional, liderazgo, creatividad, etc.) influyen de forma significativa en el grado de felicidad que declaran los individuos en las encuestas que participan como entrevistados (ej. Mroueh \& De Waal, 2020; Díaz-Pincheira\&Carrasco-Garcés,2019; Rodríguez-Pose \& Von Berlepsch, 2014; Ahn \& Mochón, 2010; Fisher,2010). En este sentido cabe señalar la ebullición en los últimos lustros del siglo XXI de una gran cantidad de estudios sobre este determinado tópico, caracterizados por usar técnicas de panel de datos para demostrar sus diferentes hipótesis de investigación (ej. Ding et al., 2020; Hendriks et al., 2020; Liu et al., 2020). Muchas de estas producciones se singularizan por emplear la palabra felicidad como sinónimos de bienestar, afecto positivo o satisfacción con la vida, indistintamente (Dominko \& Verbič,2019; Easterlin, 2001). Sin lugar a dudas, dichos trabajos han contribuido activamente a que actualmente el mundo académico posea un gran conocimiento científico sobre los vectores que tienen consecuencias directas sobre la felicidad individual de los seres humanos (Frey, 2020).

Sin embargo, los efectos que genera el parámetro voluntariado sobre la felicidad de las personas no se han explorado con gran profundidad en la sociedad digital. Esta atención limitada por parte de los investigadores está cambiando poco a poco gracias a los trabajos de Lee (2019), Magnani \& Zhu (2018), Binde \& Freytag (2013), entre otros. En ellos se evidencia empíricamente que las tareas de voluntariado que desarrollan cotidianamente los ciudadanos en las ONGDs contribuyen no solo en incrementar su estado de felicidad individual, sino además en conseguir una sociedad más solidaria, altruista y sostenible. Este fenómeno se debe básicamente a que los voluntarios asocian bienestar subjetivo o felicidad con emociones positivas. Este hallazgo, como cualquier otro, exige ir más allá en su conocimiento, en este caso concreto, podrá ser averiguar si las iniciativas de marketing social que implementen las entidades sin ánimo de lucro a través de sus redes sociales puede ayudar en avivar o fortalecer el bienestar subjetivo e interés de los voluntarios de las ONGDs a través de los principios rectores del Happiness Management (Chumaceiro-Hernández et al.,2020).

La literatura muestra que el marketing social no busca beneficios económicos necesariamente (Jiménez-Marín et al., 2019), sino que persigue cambiar de forma voluntaria la conducta de las personas con la finalidad de mejorar su bienestar y calidad de vida (Lee \& Kotler,2020). Al hilo de lo comentado, esta disciplina académica puede jugar un papel muy relevante para que los comportamientos de la ciudadanía se basen en sus emociones positivas y, por ende, en su felicidad. Las organizaciones deberían focalizar sus esfuerzos en lograr comprender el uso de las redes sociales digitales (RSD), esto les dará la posibilidad de ofrecer a los públicos contenido interesante, lo que favorecería una mayor difusión y visibilidad de la información. En definitiva, las organizaciones han de trabajar en determinar cómo movilizar al público objetivo y transformar a sus partidarios en unos promotores vocales de sus causas (RománSan-Miguel \& Díaz-Cruzado, 2019; Dooley et al. 2012; Bernhardt et al. 2012; Kotler, 2011). En las ONG los mensajes en las RSD se pueden clasificar según la dimensión denominada "eje participativo" en dos categorías: referencial o conversacional. Referencial cuando el mensaje se limita a ofrecer información y conversacional si fomenta la actividad de la audiencia (Iranzo \& Farné, 2014). Otras clasificaciones similares fueron las llevadas a cabo por Saxton \& Guo (2014) y Saxton \& Waters (2014), quienes diferencian entre mensajes informativos, 
conversacionales o de acción. Los primeros a su vez se dividen en dos clases: los denominados de 'educación pública' (Elías, 2018), que representan la información referente a posibles cambios en las políticas públicas para mejorar los intereses de los ciudadanos, y los llamados de 'marketing', que destacan por tener un tono más emocional. Según los investigadores anteriores, los de 'educación pública' generan más impacto.

Desde este enfoque, el marketing social puede constituir un potencial inductor muy válido para impulsar la construcción de una sociedad cimentada en los principios rectores de la ética, la responsabilidad social, la integridad y la sostenibilidad (Díaz-Meneses \& Basil, 2019).

\section{METOdOLOGÍA}

En esta investigación se presenta un estudio sobre el uso de Facebook por parte de las ONGDs y su relación con la felicidad de los destinatarios de los mensajes emitidos en esta red social, con especial referencia a las publicaciones sobre voluntariado. Se ha seleccionado la red social Facebook por dos motivos; por un lado, esta red social se ubica en primer lugar reconociéndose como la red presente en la mente de los internautas de forma espontánea y es utilizada por un $87 \%$ de los usuarios en nuestro país (IAB Spain, 2019); y por otro lado viene siendo la más utilizada por las Organizaciones No Gubernamentales (ONGs), en este sentido es preciso comentar que desde 2012 estudios como el de la Fundación Luis Vives comprobaron que un $94 \%$ de las citadas organizaciones utilizaban Facebook. Además de las 78 ONGDs inscritas en la Coordinadora de Organizaciones No Gubernamentales para el Desarrollo en España (CONGDE) el 99\% publican mensajes en dicha red social. Antes de comenzar con la etapa descriptiva se inició una fase exploratoria mediante una revisión de la literatura para delimitar con más precisión las cuestiones a tratar que son, la felicidad en el voluntariado, y la aplicación de las redes sociales por las ONGDs como instrumentos de marketing social en las organizaciones objeto de estudio. Las ONGDs se han seleccionado si cumplen con los siguientes requisitos:

- En primer lugar, han de formar parte de la CONGDE. La Coordinadora es la red estatal de organizaciones y plataformas sociales que trabajan en el ámbito del desarrollo, la solidaridad internacional, la acción humanitaria, la educación para la ciudadanía global y la defensa de los derechos humanos (CONGDE, 2020).

- En segundo lugar, han de estar registradas en la Agencia Española de Cooperación Internacional para el Desarrollo (AECID), el principal órgano de gestión de la Cooperación Española, orientada a la lucha contra la pobreza y al desarrollo humano sostenible. Esto supone una garantía en la transparencia de las ONGDs socias, ya que el hecho de pertenecer al citado registro obliga a verificar la idoneidad de las entidades que se inscriben, así como la veracidad de los datos inscritos, y a tomar las medidas necesarias para que la información recogida se mantenga actualizada, consiguiendo el más alto nivel de fidelidad a la realidad. En tercer y último lugar han de tener activa una página de Facebook en la que publiquen las actividades que llevan a cabo u otro tipo de información de interés para los usuarios.

En relación al alcance de la investigación es preciso comentar que se trata de un estudio descriptivo y correlacional. Esta decisión ha sido motivada por dos razones: por un lado se describe la utilización de Facebook como herramienta de marketing social, considerando como 
eje principal los mensajes que hablan de voluntariado y felicidad. En este sentido, es preciso indicar que los datos recopilados se han de convertir en información útil para la investigación, de ahí la importancia de la aplicación de técnicas de investigación descriptivas que permitan sistematizar estos datos en información fácilmente manejable y comprensible (Tinto 2013). Y por otro lado, se asocia el impacto de las publicaciones (medido por el número de likes y comentarios) con dos variables: emociones positivas y voluntariado en el mensaje, llevando a cabo un análisis correlacional bajo el planteamiento de las siguientes hipótesis sometidas a prueba.

H1: Existe relación entre el no de likes y el hecho de que los mensajes hablan de emociones positivas.

H2: Existe relación entre el no de likes y el hecho de que los mensajes hablan de emociones positivas y voluntariado.

Las características específicas de los datos proveniente de los medios sociales hacen que se requiera de una metodología específica de análisis. Para ello se ha utilizado una combinación de técnicas de minería de datos y análisis de contenido, tanto desde un enfoque cualitativo como cuantitativo. Esta metodología no solo ha de centrarse en extraer datos, sino que ha de permitir su preparación y análisis para conseguir información útil y relevante para dar respuesta a los objetivos planteados previamente, lo que se conoce como KDD-Knowlede Discovery in Databases. Este concepto se define como un proceso organizado de identificación válida, novedosa y útil que genera patrones comprensibles de los conjuntos de datos grandes y complejos (Maimon \& Rokach, 2010).

Respecto del análisis de contenido, se ha considerado como unidad de análisis el mensaje público en Facebook de cada organización, considerando como marco temporal el año 2019. En este sentido se han recopilado 3.959 publicaciones de las organizaciones con el programa Fanpage Karma y los análisis se han llevado a cabo con el software SPSS versión 24. Para la codificación se han tenido en cuenta las siguientes variables: (v1) ONGD (ver Tabla 1), (v2) № de mensajes publicados, (v2) no de likes, (v3) № de comentarios, (v4) emociones positivas (feliz, felicidad, bienestar, satisfacción, gracias, alegría, confianza, mejor, amigo/a, amor), (v5) Emoción en los mensajes (sí/no), (v6) voluntariado (sí/no) y (v7) marketing social (información, conversación y comportamiento). En relación a las categorías de emociones positivas se han considerado las investigaciones de Plutchick (2001) y Ekman (2003).

\section{RESULTADOS}

A continuación, se van a presentar los resultados agrupados en dos bloques. En primer lugar, los resultados sobre el no de mensajes publicados por las ONGDs, así como el promedio de likes y comentarios por publicación. El segundo bloque se corresponde con los resultados sobre los mensajes que hablan de emociones positivas, voluntariado y organizaciones, y en el tercer bloque se incluye la perspectiva de marketing social en los mensajes que hablan de voluntariado y emociones positivas.

En referencia al primer bloque de resultados se van a especificar de forma resumida los datos generales de la información recabada. Se han recopilado un total de 3.958 mensajes durante el año 2019 procedentes de las 10 páginas de Facebook de las ONGDs registradas en la CONGDE con mayor número de likes. En la Tabla 1 se resume la información sobre las publicaciones en 
Facebook de las organizaciones que han conseguido los mayores promedios de likes y comentarios por mensaje durante 2019. Según los resultados, Mensajeros de la Paz España es la ONGD con mayor promedio de likes por mensaje. Es preciso comentar que la mayoría de estas publicaciones son de tipo religioso o informativo (mostrando donaciones que han conseguido de diversas empresas, particulares o celebridades).

Tabla 1

Datos referentes a los mensajes publicados en Facebook por las ONGDs con mayor promedio de likes durante 2019

\begin{tabular}{|c|c|c|c|}
\hline ONGDs & № de mensajes publicados & $\begin{array}{l}\text { Promedio de } \\
\text { likes/mensaje }\end{array}$ & $\begin{array}{l}\text { Promedio de } \\
\text { comentarios/mensaj } \\
\text { e }\end{array}$ \\
\hline Mensajeros de la Paz España & 704 & 114,05 & 7,13 \\
\hline Cruz Roja Española & 509 & 103,46 & 4,40 \\
\hline Cáritas Española & 280 & 80,39 & 1,54 \\
\hline Oxfam Intermón & 469 & 75,35 & 30,04 \\
\hline Manos Unidas & 352 & 72,61 & 1,21 \\
\hline Movimiento por la Paz - MPDL & 101 & 60,64 & 0,85 \\
\hline Amigos de la Tierra & 408 & 55,50 & 3,59 \\
\hline Médicos del Mundo España & 288 & 40,98 & 3,25 \\
\hline Entreculturas & 747 & 33,42 & 0,95 \\
\hline AIDA, Ayuda, Intercambio y Desarrollo & 100 & 30,89 & 1,61 \\
\hline Total & 3.958 & 72,00 & 6,46 \\
\hline
\end{tabular}

En referencia a los mensajes que han conseguido más "me gusta", es interesante señalar dos publicaciones: una de Cruz Roja que hace referencia al apoyo a las personas con movilidad reducida para que puedan pasear por la playa, y otra, la que ha obtenido el mayor número de likes de las 45 ONGDs, un mensaje de Oxfam Intermón, del que es preciso destacar que no contiene ningún vocablo relacionado con emociones positivas. Es un mensaje de abril de 2019 referente a las elecciones y trata sobre la pobreza. Su principal característica es que incita a la conversación. El texto es el siguiente:

"Las Elecciones Generales están a la vuelta de la esquina. Un dato: en España hay casi 10 millones de personas que viven bajo el umbral de la pobreza. De ellos, 2,3 millones son niños y niñas. ¿Cómo solucionarías esto? Nos interesa saber qué opinas. \#MenudaPapeleta \#ReducirlaDesigualdad"

A continuación, se presentan los resultados del segundo escenario o bloque, sobre los mensajes que hablan sobre emociones positivas. En relación a los mensajes publicados por las organizaciones objeto de estudio y después de haber seleccionado aquellos que contienen las palabras relacionadas con emociones positivas (especificadas en el apartado de metodología), es preciso comentar que la mayor parte de las publicaciones se refieren a mensajes de agradecimiento, seguidas de las que contienen los vocablos "mejor" y "amigo/a". El resto de los términos no han alcanzado el $10 \%$ en el recuento de las palabras más recurrentes, como se muestra en la Figura 1. 


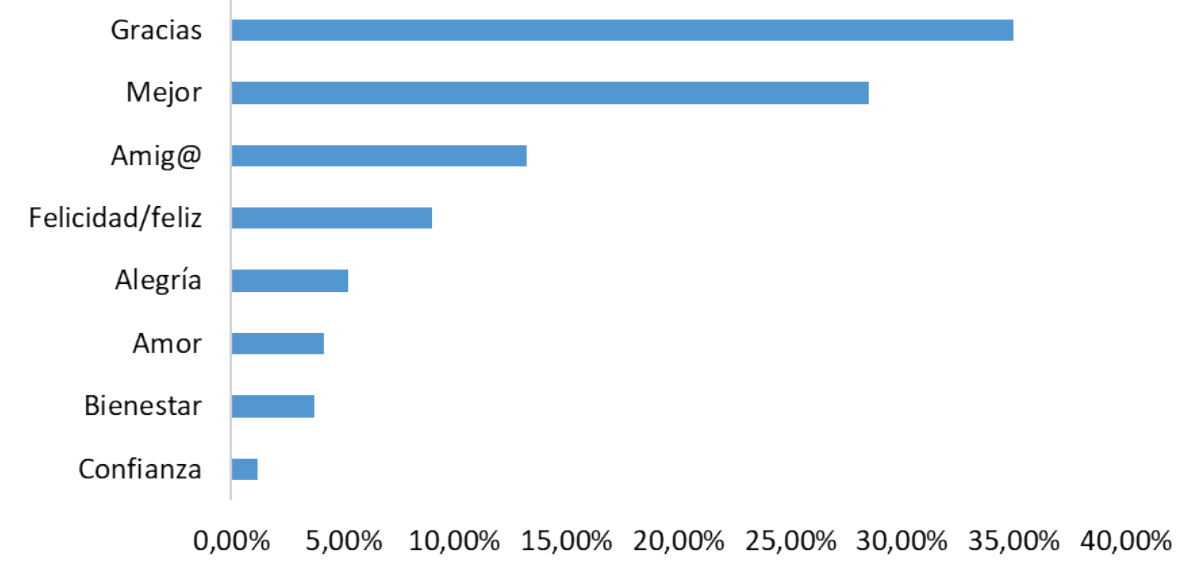

Figura 1. Términos relacionados con emociones positivas más publicados por las ONGDs objeto de estudio durante 2019

Llegados a este punto resultaba interesante comprobar cuál es el interés de los usuarios por los mensajes que contienen los términos especificados en la figura 2. Para ello se han elaborado las Figuras 3 y 4 , que muestran tanto el número de mensajes publicados con los vocablos de emociones positivas, así como el promedio de likes y comentarios (por mensaje) respecto cada organización, respectivamente. El número total de mensajes publicados con vocablos sobre emociones positivas es de 915. En la Figura 3 se muestra el porcentaje de dichos mensajes, siendo Mensajeros de la Paz España la organización que ha emitido más publicaciones con este tipo de palabras. Es destacable el hecho de que en ninguna de las ONGDs analizadas, excepto la mencionada anteriormente, el porcentaje no alcance el $20 \%$.

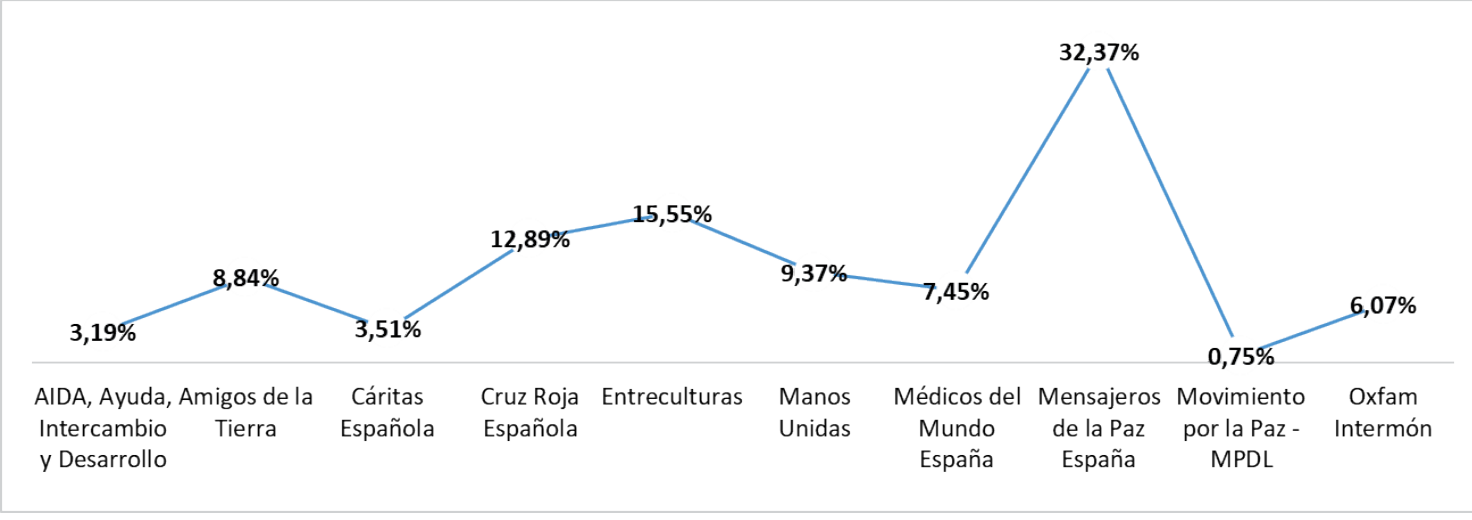

Figura 2. № mensajes publicados que hablan sobre emociones positivas 

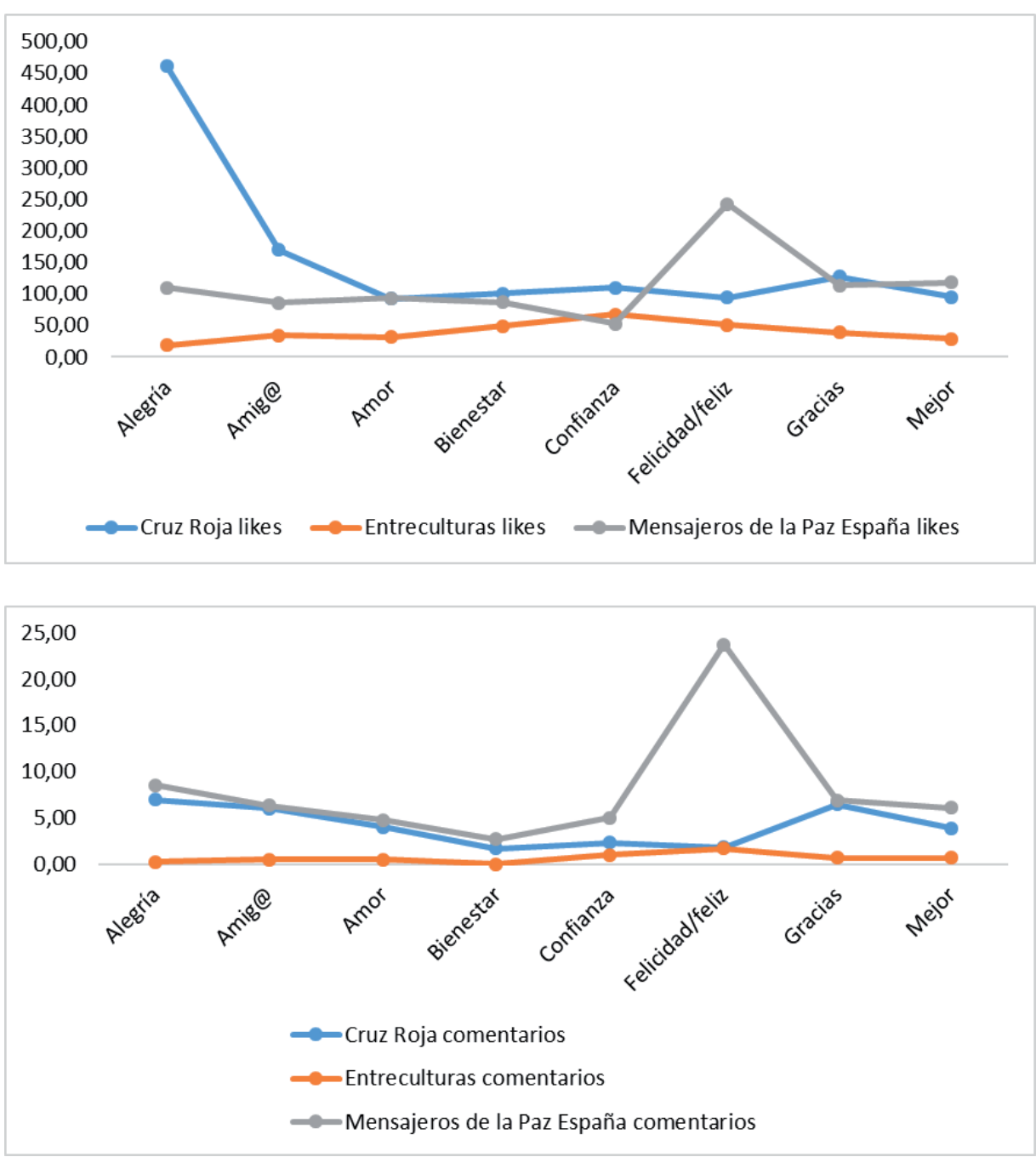

Figura 3. Promedio de me likes y comentarios de las publicaciones que hablan sobre emociones positivas

En la Figura 3 se han seleccionado las organizaciones con más mensajes publicados sobre emociones positivas. El mayor no de likes se ha obtenido en una publicación de Cruz Roja que habla sobre la alegría y la esperanza de un niño que vuelve a bailar con una prótesis recibida gracias a la ONGD. Por otra parte, el vocablo felicidad es uno de los que ha suscitado el mayor interés del público tanto por el no de likes y comentarios.

Una vez realizado este apunte se exponen los resultados sobre los mensajes que hablan de voluntariado y emociones positivas. Es preciso comentar que, a pesar de ser un colectivo de suma importancia para las ONGDs, no había una proporción muy elevada de publicaciones que mencionasen la palabra voluntario, voluntaria o voluntariado, en concreto un $23,12 \%$. Si de estos mensajes se consideran los que hablan de emociones positivas el porcentaje desciende a un 10,92\%. En la Figura 4 se puede observar cuántos de los mensajes sobre voluntariado contienen emociones positivas. 


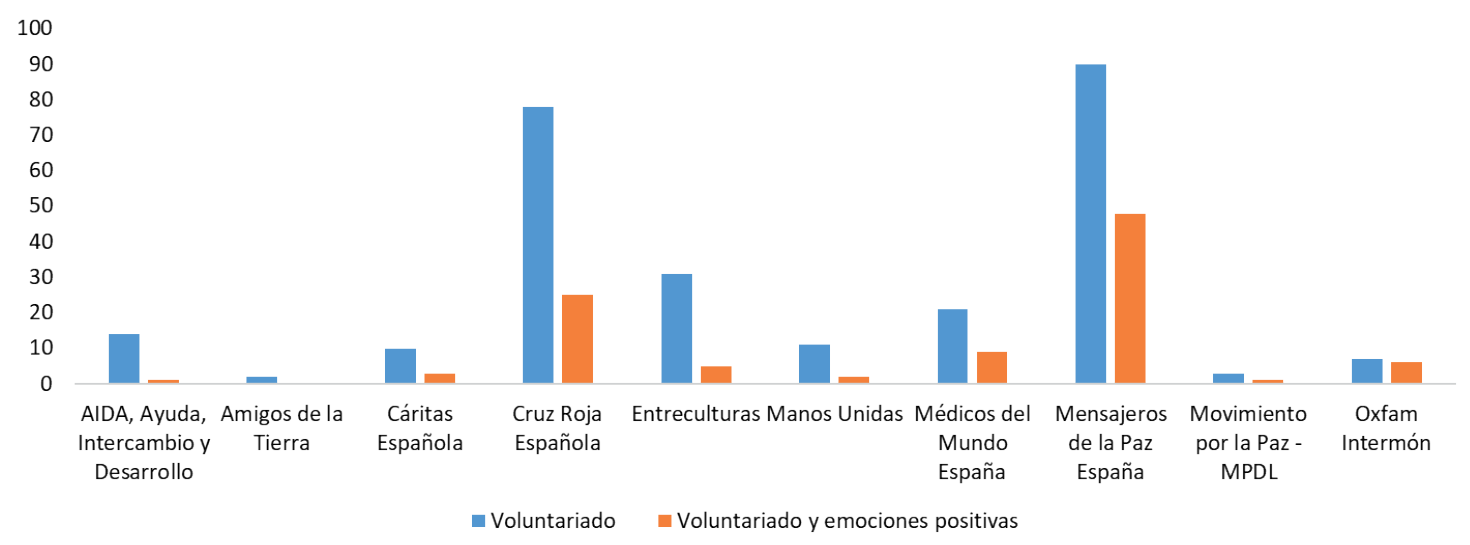

Figura 4. № de mensajes que hablan de voluntariado y no de publicaciones sobre voluntariado y emociones positivas

En la figura 5 se presenta el promedio de likes y comentarios de las emociones positivas en los mensajes que hablan sobre voluntariado.
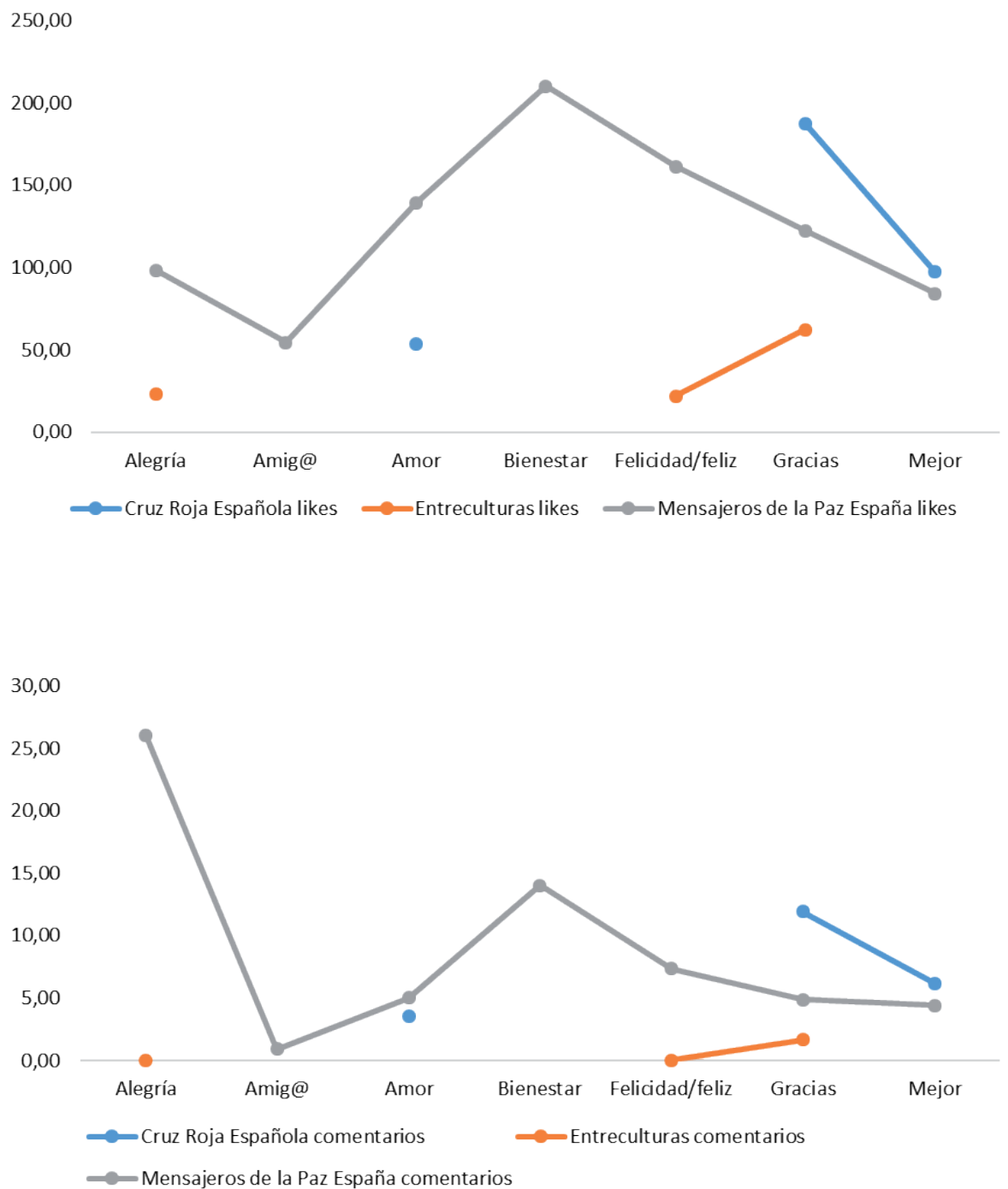


\section{Figura 5. Promedio de likes y comentarios de las publicaciones que hablan sobre voluntariado y emociones positivas}

A tenor de estos resultados cabe preguntarse ¿Qué características, desde la perspectiva del marketing social, tienen los mensajes sobre voluntariado y emociones positivas que han conseguido el mayor número de likes? ¿Existe relación entre el promedio de likes del mensaje con el hecho de que hable sobre voluntariado y emociones positivas?

Examinando las publicaciones se ha observado que Cruz Roja Española es la ONGD qué más publicaciones sobre voluntariado emite. También es la que ha publicado más mensajes de tipo comportamiento, es decir, posts que animan a colaborar, bien donando (alimentos, dinero, etc.), ayudando a hacer más virales los mensajes en las redes sociales, participando como voluntario o asistiendo a algún evento. Aunque la publicación que ha tenido más impacto es una de Cruz Roja de agradecimiento, que ha conseguido 914 likes. En el mensaje aparece la palabra gracias en dos ocasiones y también el término disfrutar. Destaca su contenido, que refleja en un vídeo cómo las personas con movilidad reducida pueden disfrutar de la playa con la ayuda de los voluntarios. El mensaje es el siguiente:

Un año más, jestamos \#CadaVeranoCercadeTi! Pepita es una de las personas que puede disfrutar de la playa gracias al servicio de \#bañoadaptado de Cruz Roja. Este servicio, que permite a las personas con movilidad reducida disfrutar de un baño en el mar, está presente en más de 60 playas del litoral. Cruz Roja Española - Provincia de Alicante nos acerca la historia de Pepita. ¡Muchas gracias a todo el personal voluntario por hacer que muchas personas como Pepita puedan disfrutar del verano! (https://www.facebook.com/CruzRoja.es/posts/10162230963735158/)

El segundo mensaje con más impacto ha sido uno de tipo religioso de Mensajeros de la Paz España sobre la inauguración de una iglesia en Roma en la que estuvo su presidente, el Padre Ángel. En este mensaje se reconoció la labor de manera personalizada de algunos voluntarios, nombrándolos en la publicación.

Desde el punto de vista del marketing social es de destacar que la mayoría de los mensajes que hablan sobre voluntariado y emociones positivas al mismo tiempo son de tipo conversacional, es decir, incitan al diálogo. Esto puede ser debido a la naturaleza de los vocablos relacionados con la felicidad, como por ejemplo el término gracias. Muchos de los mensajes que contienen la palabra 'gracias' hacen referencia a historias personales, bien de beneficiarios o voluntarios/as. También se agradece la labor de grupos concretos de voluntarios que han sido designados para un trabajo específico, o animan a la colaboración. Los mensajes de comportamiento son menos numerosos (18\%) y los de información representan el 31\%. Los mensajes de comportamiento hacen referencia a participar en eventos, visitar la página web de la organización u otros más concretos que piden voluntarios sanitarios o para colaborar en verano, como el siguiente mensaje de Mensajeros de la Paz España.

\section{URGENTE/NECESITAMOS VOLUNTARIOS PARA LOS DESAYUNOS, CENAS Y TELEFONO DORADO}

En verano necesitamos voluntarios/as para poder seguir apoyando a las personas más vulnerables. Si estás interesado/a en colaborar en los desayunos en la iglesia de SAN 
ANTON, las cenas en los restaurantes solidarios ROBIN HOOD y acompañado a las personas en soledad a través de nuestro TELÉFONO DORADO. No dudes en llamarnos de lunes a viernes de $9 \mathrm{~h}$ a $13 \mathrm{~h}$ al teléfono 9002222 23. Si conoces a alguien al que le puede interesar mensaje iiimándaselo!!! (https://www.facebook.com/mensajerosdelapazespana/posts/2101257336805290/)

Los mensajes de información son los que han conseguido un promedio de likes por mensaje mayor $(123,23)$, esto puede ser debido a que hay mensajes de agradecimiento que al hablar de forma impersonal sobre una acción lograda se han catalogado como informativos.

En respuesta a la segunda y tercera pregunta, los resultados de la prueba de Kruskal Wallis mostraron: en relación a la hipótesis 1 , no existen diferencias significativas entre el número de likes y el hecho de que los mensajes hablen de emociones positivas ( $p>0,05)$, y, en relación a la hipótesis 2, sí existen diferencias significativas entre el número de likes y los mensajes que hablan sobre emociones positivas y voluntariado $(p<0,05)$. Esto puede comprobarse si consideramos el valor del mencionado indicador por cada vocablo de felicidad. Los mensajes con mayor valor promedio de likes han sido los que contienen la palabra disfrutar, esto ha sido debido a un solo mensaje de Cruz Roja (especificado anteriormente), que ha conseguido 914 likes. Otro dato a destacar es que el vocablo 'gracias' ha sido uno de los más utilizados (figura 1) aunque los mensajes que contienen esta palabra han conseguido un promedio de 39,32 likes. Sin embargo, si tenemos en cuenta cada ONGD la situación varía, es decir, en la mayoría de las organizaciones los mensajes que contienen la palabra 'gracias' han sido los más exitosos.

\section{Tabla 2}

Pruebas de Kruskal Wallis

Estadisticos de prueba $a^{a, b}$

\begin{tabular}{|l|r|}
\hline a,b & \multicolumn{1}{|c|}{ Likes } \\
\hline Chi-cuadrado & 7,050 \\
gl & 6 \\
Sig. asintótica &, 316 \\
\hline
\end{tabular}

a. Prueba de Kruskal Wallis

b. Variable de agrupación: Emoción positiva
Estadisticos de prueba ${ }^{a, b}$

\begin{tabular}{|l|r|}
\hline a,b & \multicolumn{2}{|c|}{ Likes } \\
\hline Chi-cuadrado & 64,726 \\
gl & 1 \\
Sig. asintótica &, 000 \\
\hline
\end{tabular}

a. Prueba de Kruskal Wallis

b. Variable de agrupación: Emoción positiva y voluntariado

\section{CONCLUSIONES}

En este trabajo científico se ha estudiado cuál es el impacto de los mensajes (medido por el número de likes y comentarios) publicados por las ONGDs objeto de estudio, focalizando el análisis en aquellos posts que hablan sobre voluntariado y emociones positivas, y añadiendo la perspectiva de marketing social. Resulta destacable que aquellas publicaciones que contienen los vocablos 'gracias', 'bienestar' y 'alegría' son los que han obtenido un mayor promedio de likes y comentarios, destacando en este sentido las publicaciones de Cruz Roja España. A este respecto es preciso comentar que, coincidiendo con las investigaciones de Campbell \& Lambright (2020), Saxton \& Waters (2014) o Lovejoy \& Saxton (2014), los mensajes de agradecimiento son los más efectivos en los perfiles de Facebook de las ONGs. Sin embargo, es preciso comentar a este respecto que, en la mayoría de los mensajes analizados, las ONGDs no nombran a los voluntarios, sobre todo en organizaciones tan populares como Cruz Roja y Cáritas. Sin embargo, estos han obtenido un mayor promedio de likes que los mensajes que no 
hablan sobre este colectivo tan importante para este tipo de organizaciones. Quizás pueda ser debido al alcance o número de seguidores de las citadas organizaciones, por lo que dicha variable podría incluirse en futuras investigaciones.

Por otra parte, es preciso destacar dos resultados interesantes. Por un lado, hay evidencias para afirmar la existencia de una relación entre los likes y el hecho de que aparezcan los vocablos referentes a emociones positivas, siendo mayor el impacto en el caso de los mensajes que contienen los términos relacionados con la felicidad. $Y$ por otro lado, no se han encontrado evidencias suficientes para afirmar que hay relación entre el no de likes y el tipo de vocablo sobre emociones positivas que contenga el mensaje. De esto se deduce que existen otros motivos que dan lugar a que los usuarios tengan más o menos interés en las publicaciones, que pueden ser el contenido del mensaje y el formato multimedia (fotos, imágenes o vídeos). Este último elemento, que hace más atractivas las publicaciones en los medios sociales, obliga a las entidades sociales a actualizar continuamente sus contenidos con el objeto de avivar la reacción del público. Para Guidry, Waters \& Saxton (2014) esta dinamización de la actividad dará lugar a que los usuarios argumenten y compartan la información aumentando así los niveles de participación de los interesados.

Respecto a las características de los mensajes desde una perspectiva de marketing social destacan los siguientes aspectos. Por un lado, la mayoría de las publicaciones contienen el vocablo 'gracias', estos son mensajes de agradecimiento. Sin embargo, en este estudio se ha encontrado un matiz en este tipo de publicaciones. Los mensajes que contienen la palabra 'gracias' son de dos clases, una en la que se informa de manera impersonal sobre la labor realizada por los voluntarios, este tipo de mensajes han sido clasificados en esta investigación como informativos; y otra, en la que se enfatiza la identidad de los voluntarios o grupo de voluntarios reconociendo el trabajo específico que llevaron a cabo. Este tipo de mensajes es el que se ha catalogado como de conversación. También destacan entre los mensajes de conversación los que cuentan historias de voluntarios o personas que han conseguido superar importantes dificultades que les han llevado a una situación de exclusión social, y por último también son comunes en algunas organizaciones los mensajes de tipo religioso. Sobre este punto, Muñoz (2015) indica que es necesario concebir la comunicación como un diálogo, es decir, ser más conversadores que informadores. Esto requiere de un entorno favorable hacia la participación para fomentar la voluntad de interactuar con individuos de diversas opiniones, cultivándose de esta manera la confianza entre las partes (Kenty Taylor, 2002). También es de destacar que las ONG en general son propensas a emplear las RSD como canales de publicidad tradicionales, esto implica que son meros promotores de sus actuaciones, es decir, no aprovechan la oportunidad que ofrece dicho medio para impulsar el diálogo on line (Ramil,2012). Esta idea coincide con la de Arroyo, Baños\& Rodríguez (2012) quienes afirman que las ONG cuentan en los mensajes lo que hacen o falta por hacer. Continuando con las características de los mensajes desde la perspectiva del marketing social es preciso comentar que en este estudio los mensajes de comportamiento son escasos y no han conseguido un importante impacto, esto puede dar pie a que futuras investigaciones se focalicen en este tipo de publicaciones y su relación con los comportamientos solicitados en el entorno off line.

Desde la perspectiva del marketing social todo lo comentado hasta aquí cobra especial sentido en el entorno on line pues lo que se pretende es movilizar al público con el objeto de que sea 
defensor vocal de las causas sociales de las organizaciones. Esto puede conseguirse incluyendo vocablos relacionados con la felicidad en los mensajes.

\section{Referencias}

Ahn, N. \& Mochón, F. (2010). La felicidad de los españoles: factores explicativos. Revista de economía aplicada, 18(54), 5-31.

Andreasen A. (1994). Social Marketing: Its Definition and Domain. Journal of Public Policy \& Marketing, 13(1), 108-114.

Arroyo, l.; Baños, M. \& Rodríguez, T. (2012). Modelo de investigación de las piezas audiovisuales de las organizaciones del tercer sector en redes sociales. Tarragona, actas del congreso AE-IC, 1-18.

Ashtiani, A. Z.; Dudek, T. \& Rieger, M. O. (2020). Happy savers and happy spenders: An experimental study comparing US Americans and Germans. Journal of Behavioral and Experimental Economics, 85, 101506. https://doi.org/10.1016/j.socec.2019.101506

Bakker, A.; Burger, M., van Haren, P., Oerlemans, W., \& Veenhoven, R. (2020). Raise of Happiness Following Raised Awareness of How Happy One Feels: A Follow-Up of Repeated Users of the Happiness Indicator Website. International Journal of Applied Positive Psychology https://doi.org/10.1007/s41042-020-00032-w

Bernhardt, J.; Mays, D. \& Hall, A. (2012). Social marketing at the right place and right time with new media. Journal of Social Marketing, 2(2), 130-137.

Binder, M.; \& Freytag, A. (2013). Volunteering, subjective well-being and public policy. Journal of Economic Psychology, 34, 97-119. https://doi.org/10.1016/j.joep.2012.11.008

Borgonovi, F. (2008). Doing well by doing good. The relationship between formal volunteering and selfreported health and happiness. Social science \& medicine, 66(11), 2321-2334. https://doi.org/10.1016/j.socscimed.2008.01.011.

Chumaceiro-Hernández, A.C.; Hernández- G.de Velazco, J.J.; Ravina-Ripoll, R. \& Reyes-Hernández, I.V. (2020) University Social Responsibility in the Organizational Happiness Management. Utopia y Praxis Latinoamericana, 25(2), 427-440

Campbell, D. A. \& Lambright, K. T. (2020). Terms of engagement: Facebook and twitter use among nonprofit human service organizations. Nonprofit Management and Leadership, 30(4), 545-568. doi:10.1002/nml.21403

Coordinadora de Ong para el Desarrollo España (CONGDE) (10 de julio de 2020). Nuestra historia. Recuperado de https://coordinadoraongd.org/coordinadora/historia-y-logros/.

Crespo, R. F. \& Mesurado, B. (2015). Happiness economics, eudaimonia and positive psychology: From happiness economics to flourishing economics. Journal of Happiness Studies, 16(4), 931-946. https://doi.org/10.1007/s10902-014-9541-4

Dann S. (2010). Redefining social marketing with contermporary commercial marketing definitions. Journal of Business Research, 63, 147-153.

Díaz-Meneses, G. \& Basil, MD (2019). A birief History of Social Marketinl. In Basil D.; Diaz-Meneses G. \& Basil M. (Eds). Social Marketing in Action. Springer Texts in Business and Economics Social Marketing in Action (pp. 79-89). https://doi.org/10.1007/978-3-030-13020-6_5

Díaz-Pincheira, F. J. \& Carrasco- Garcés, M. E. (2019). Effects of organizational climate and psychosocial risks on happiness at work. Contaduría y Administración, 63, 114.http://dx.doi.org/10.22201/fca.24488410e.2018.1142

Ding, J.; Salinas-Jiménez, J. \& Salinas-Jiménez, M. D. M. (2020). The Impact of Income Inequality on Subjective Well-Being: The Case of China. Journal of Happiness Studies, 1-22. https://doi.org/10.1007/s10902-020-00254-4

Dooley, J.; Jones, S. \& Iverson, D. (2012). Web 2.0: an assessment of social marketing principles. Journal 
of Social Marketing, 2(3), 207-221.

Dominko, M. \& Verbič, M. (2019). The economics of subjective well-being: A bibliometric analysis. Journal of Happiness Studies, 20(6), 19731994.https://doi.org/10.1007/s10902-018-0022-z

Easterlin, R. A. (2001). Income and happiness: Towards a unified theory. The Economic Journal, 111(473), 465-484. https://doi.org/10.1111/1468-0297.00646

Ekman, P. (2003). Emotions Revealed. Times Books.

Elías Zambrano, R. (2018). Digital advertising storytelling: consumer educommunication. IROCAMM International Review of Communication and Marketing Mix, 1, 32-44. http://dx.doi.org/10.12795/IROCAMM.2018.i1.02

Fisher, C. D. (2010). Happiness at work. International Journal of Management Reviews, 12(4), 384-412. https://doi.org/10.1111/j.1468-2370.2009.00270.x.

Frey, B.S. (2020). What are the opportunities for future happiness research? International Review of Economics, 67, 5-12. https://doi.org/10.1007/s12232-019-00318-9

French J. \& Blair-Stevens C. (2006). From snake oil salesmen to trusted policy advisors: the development of a strategic approach to the application of social marketing in England.Social Marketing Quarterly, 12(3), 29-40.

Fundación Luis Vives (2012). Uso de las redes sociales en las ONG españolas. https://www.telefonodelaesperanza.org/noticias/view/5462

Guidry, J.; Waters, R. \& Saxton, G. (2014). Moving social marketing beyond personal change to social changeStrategically using Twitter to mobilizesupporters into vocal advocates. Journal of Social Marketing, 4(2), 40-260.

Hendriks, M., Burger, M., Rijsenbilt, A., Pleeging, E., \& Commandeur, H. (2020). Virtuous leadership: a source of employee well-being and trust. Management Research Review. 43(8), 951-970. https://doi.org/10.1108/MRR-07-2019-0326

IAB Spain; Elogia (2019). Estudio Anual de Redes Sociales 2019. Recuperado de https://iabspain.es/estudio/estudio-anual-de-redes-sociales-2019/

Iranzo A. \& Farné, A. (2014). Herramientas de Comunicación para el Tercer Sector: El uso de las Redes Sociales por las ONGD Catalanas. COMMONS - Revista de Comunicación y Ciudadanía Digital 3, (2), 29-50.

Jiménez-Marín, G.; Elías Zambrano, R.; Bellido-Pérez, E. \& Sanz-Marcos, P. (2019). Eficacia del endorsement marketing: La comunicación de las empresas de retail a través de Instagram. En Sierra Sánchez, J. \& Lavín de la Cavada, J. M. (eds.). Redes sociales, tecnologías digitales y narrativas interactivas en la sociedad de la información, 109-120. Madrid: McGraw-Hill.

Kent, M. \& Taylor, M. (2002). Toward a dialogic theory of public relations. Public Relations Review, 28, 21-37.

Kotler, P. (2011). Reinventing marketing to manage the environmental imperative. Journal of Marketing, 75, 132-135.

Lee, N. R. \& Kotler, P. (2020). Social marketing: behavior change for social good. California, USA: SAGE Publications.

Lee, M. A. (2019). Volunteering and happiness: Examining the differential effects of volunteering types according to household income. Journal of Happiness Studies, 20(3), 795-814. https://doi.org/10.1007/s10902-018-9968-0

Liu, Z.; Zhong, X.; Zhang, T. \& Li, W. (2020). Household debt and happiness: evidence from the China Household Finance Survey. Applied Economics Letters, 27(3), 199-205. https://doi.org/10.1080/13504851.2019.1610706

Magnani, E. \& Zhu, R. (2018). Does kindness lead to happiness? Voluntary activities and subjective wellbeing. Journal of behavioral and experimental economics, 77, 20-28. https://doi.org/10.1016/j.socec.2018.09.009 
Maimon, O. \& Rokach, L. (2010). Data Mining and Knowledge Discovery Handbook. Cham: Springer.

Mroueh, M. \& De Waal, A. (2020), Measuring happiness at work in a Takaful organization, Journal of Organizational Effectiveness: People and Performance, https://doi.org/10.1108/JOEPP-04-20200050

Muñoz, I. (2015). El alcance mediático de los gabinetes de comunicación de las ONGD pro inmigrantes. Análisis de la campaña ¿Y tú de quién eres? De la fundación Sevilla Acoge en 2013. (tesis doctoral). Facultad de Comunicación. Universidad de Sevilla.

Plutchik, R. (2001). The Nature of Emotions: Human emotions have deep evolutionary roots, a fact that may explain their complexity and provide tools for clinical practice. American Scientist, 89(4), 344-350.

Ramil, X. (2012). ¿Cuál es el nuevo paradigma de la comunicación en el que nos movemos las ONG de Desarrollo? En Ramil, Xosé (coordinación): ParadigmáTIC@s, Comunicación y Cultura Digital en las ONGs de Desarrollo.

Ravina-Ripoll, R.; Tobar-Pesantez, L. \& Galiano-Coronil, A. (2018). Claves para un desarrollo sostenible La creatividad y el happiness management como portafolio de la innovación tecnológica, empresarial y marketing social. Editorial Comares.

Rodríguez-Pose, A. \& Von Berlepsch, V. (2014). Social capital and individual happiness in Europe. Journal of Happiness Studies, 15(2), 357-386.https://doi.org/10.1007/s10902-013-9426-y

Román-San-Miguel, A. \& Díaz-Cruzado, J. (2019). Communication and advertising in NGDOs: Present and future. IROCAMM: International Review of Communication and Marketing Mix, 2 (1), 95-101. 10.12795/IROCAMM.2019.v02.i01.09

Saxton, G. \& Guo, C. (2014). Tweeting Social Change: How Social Media AreChanging NonprofitAdvocacy. Nonprofit and Voluntary Sector Quarterly 2014, 43(1), 57-79.

Saxton, G. \& Waters, R. (2014). What do Stakeholders Like on Facebook? Examining Public Reactions to Nonprofit Organizations' Informational, Promotional, and Community-Building Messages. Journal of Public Relations Research, 6, 280-299.

Schwarze, J. \& Winkelmann, R. (2011). Happiness and altruism within the extended family. Journal of Population Economics, 24 (3), 1033-1051. https://doi.org/10.1007/s00148-010-0326-8

Tanaka, S. \& Tokimatsu, K. (2020). Social Capital, Subjective Well-Being, and Happiness: Evidence from a Survey in Various European and Asian Countries to Address the Stiglitz Report. Modern Economy, 11(02), 322.

Tinto, J. (2013). El análisis de contenido como herramienta de utilidad para la realización de una investigación descriptiva. Un ejemplo de aplicación práctica utilizado para conocer las investigaciones realizadas sobre la imagen de marca de España y el efecto país de origen. Provincia, 29, 135-173.

\section{Semblanza de los autores}

Araceli Galiano Coronil es doctora y profesora de Marketing en la Universidad de Cádiz, y pertenece al grupo de investigación Innovación Social en Marketing. También ha realizado estancias de investigación en las universidades de Sevilla y Granada, y ha sido profesora visitante en varias universidades como: la Universidad de Venecia, la Business and Law Frankfurt University of Applied Sciences (Frankfurt, Alemania), Degli Studi di Verona, EDHEC Business School (Lille, Francia), Fachhochschule (Trier, Alemania), Faculty of Business Augsburg University of Applied Sciences (Ausburgo, Alemania), ISM International School of Management Campus Hamburg (Hamburgo, Alemania), donde ha impartido conferencias sobre marketing social en ONGD. Es revisora de revistas como Sustainability. Además, es autora y coautora de 
artículos y ponencias en congresos internacionales sobre marketing social en entidades no lucrativas, y ha publicado en varias revistas internacionales.

Rafael Ravina Ripoll es doctor y profesor de Organización de Empresas en la Universidad de Cádiz, y miembro del Grupo de Investigación "Estudios Históricos Esteban Boutelou". Ha sido profesor visitante en la Universidad de Ciencias Aplicadas de Frankfurt y en la Universidad de Verona, donde ha impartido conferencias sobre "Gestión de la Felicidad". Es autor y coautor de artículos, trabajos en congresos internacionales y libros relacionados con la economía de la felicidad y la creatividad, y desde 2018, miembro del Comité Editorial de la Revista "Retos" de la Universidad Politécnica Salesiana de Ecuador. 